\title{
Diagnosis Model of Volleyball Skills and Tactics Based on Artificial Neural Network
}

\author{
Wei Jiang, ${ }^{1}$ Kai Zhao $\mathbb{D},{ }^{1}$ and Xinlong Jin ${ }^{2}$ \\ ${ }^{1}$ China Volleyball College, Beijing Sport University, Beijing 100089, China \\ ${ }^{2}$ Academic Affairs Office, Dalian University of Science and Technology, Dalian 116000, Liaoning, China
}

Correspondence should be addressed to Kai Zhao; paiqiu@bsu.edu.cn

Received 8 July 2021; Revised 6 August 2021; Accepted 12 August 2021; Published 21 August 2021

Academic Editor: Sang-Bing Tsai

Copyright (c) 2021 Wei Jiang et al. This is an open access article distributed under the Creative Commons Attribution License, which permits unrestricted use, distribution, and reproduction in any medium, provided the original work is properly cited.

With the development of China's sports industry, the technical and tactical level of the team is required to be higher and higher. This study mainly discusses the diagnostic model of volleyball technique and tactics based on artificial neural network. With the help of the correlation function in Matlab neural network toolbox, in the training process of volleyball technical and tactical evaluation neural network, the sample data of volleyball technical evaluation index is repeatedly simulated and studied, and finally the network parameters with the minimum error and the highest accuracy are saved as the network model for subsequent verification and evaluation. The middle layer is the hidden layer, which makes the network approach the result of volleyball experts' evaluation of the technology by adjusting the weights of neurons. The last layer is the output layer, which outputs the actual evaluation results of volleyball experts on the technology. Through repeated training and comparison of input samples, the maximum number of training times of BP network for volleyball technical and tactical evaluation is determined to be 32 . Some common experience of estimating hidden node number is provided by trial-and-error method. On this basis, the number of hidden nodes to minimize the network error is finally determined to be 4 through repeated training and comparison. In the process of network diagnosis, the average difference between the evaluation score of network output and the score of actual experts is less than $1 \%$, which reaches a very high precision. It shows that the volleyball skill evaluation model based on BP neural network is feasible in technology and the result is relatively reliable.

\section{Introduction}

The evaluation of the team's technical and tactical level is mainly achieved through the analysis of the application effect of the indicators reflecting the technical and tactical level of volleyball. Through the statistics and data processing of technical and tactical indicators in the process of competition, the most direct information can be provided for scientific training [1]. Before the game, coaches and decision makers can understand the basic situation of the team members and the whole team according to the previous technical and tactical statistical data of the team so as to formulate the team's technical and tactical play methods and so as to know yourself and the enemy and have a definite target.

In the competition scene, the coach can conduct on-thespot command through the timely feedback of the game technical and tactical analysis data so as to achieve the flexibility. The technical and tactical level of volleyball is the professional level of the whole team reflected by the application effect of the techniques and tactics in a volleyball match.

The recurrent neural network training algorithm based on the extended Kalman filter can predict the price of electric energy. Alanis and Alma also included proof of stability using the well-known Lyapunov method for the proposed artificial neural network trained using an algorithm based on the extended Kalman filter. Their research process lacked practice [2]. Daniel et al. believed that material defects in the steam generator tube (SGT) of the sodium-cooled prototype fast breeder reactor (PFBR) may cause water to leak into the sodium. They used COMSOL Multiphysics 4.3a software to model the rectangular notch defect on the outer surface of 
the steam generator tube. His research process is too complicated [3]. Carvalho et al. believed that topological invariants can represent the Hamiltonian, thereby predicting the existence of gap modes for topological protection. By combining neural networks with electronic state calculations using the nuclear polynomial method, they showed that local evaluation of invariants can be performed by evaluating local quantities. Their research was not very practical [4]. Pandey and Mishra believed that forecasting crop yields was very helpful for the food management and growth of a large economy based on agriculture. They verified that both GRNN and RBNN can accurately predict potato production. However, based on the fast learning ability and lower expansion constant (0.5), it is found that GRNN is better than RBFNN. In addition, it was found that the rough surface field is more productive than the flat field. Their research process lacked comparative experiments [5].

With the help of the relevant functions in the MATLAB neural network toolbox, in the training process of the volleyball technical and tactical evaluation neural network, the sample data of the volleyball technical evaluation index is repeatedly simulated and learned, and finally the network parameters with the smallest error and the highest accuracy are saved as subsequent verification and evaluation network model. According to research needs, four BP neural network models of volleyball serve, pad, pass, and buckle are established. The first layer of the model is the input layer, and the input variables are the evaluation indicators of this volleyball technique.

\section{Diagnosis Model of Volleyball Skills and Tactics}

2.1. Volleyball Skills and Tactics. The level of volleyball techniques and tactics is mainly reflected by the application effects of volleyball techniques and tactics, that is, mainly reflected by the application effects of representative volleyball techniques and tactics $[6,7]$. So which technical and tactical indicators can reflect the level of volleyball skills and tactics is the focus of this section [8]. By referring to the past experience in the design of volleyball technical and tactical indicators and discussing with relevant experts, the design of volleyball technical and tactical indicators in this article first starts from the perspective of volleyball technical and tactical theory and analyzes whether they can reflect the characteristics of volleyball technical and tactics. The indicators with large amount of information and strong representative were selected as preliminary indicators. The indicator design of this research is divided into two parts $[9,10]$. First, from the perspective of offense and defense, design independent indicators of offensive and defensive techniques and tactics that reflect comprehensive and concise information and are conducive to practical operations; second, under the premise of introducing the second pass, from the perspective of dynamic connection, analyze the relationship between the second level and cutting-edge technology, later technology, and design series indicators [11].
Our evaluation of volleyball offensive and defensive technical and tactical levels is mainly reflected by the application effects of representative offensive and defensive technical and tactical indicators $[12,13]$. Therefore, for the design of volleyball offensive and defensive technical and tactical indicators, we should first analyze the techniques and tactics from the offensive and defensive perspectives and choose the offensive and defensive technical and tactical indicators with strong representativeness and large amount of information as the object of evaluation [14]. First of all, from the offensive perspective, serving mainly relies on the individual player's technical and tactical abilities, and the effect of destroying the opponent's defense is achieved by means of floating, jumping, and vigorously serving. The emphasis is on the individual's technical and tactical capabilities, and this article mainly evaluates this point. The target is collective skills and tactics, so the specific use of certain tactics to serve is not considered; only the effect of the serve is considered. Smashing is mainly based on the changeable setter tactics in the practice game, and there are different ways of playing $[15,16]$. Therefore, spiking reflects not only technical information but also tactical information [17]. From the perspective of reflecting the amount of information, considering only the spike, the information reflected is relatively vague and general, which is of little significance to the coaches in analyzing the skills and tactics of the team. However, if the smash is divided very carefully, such as considering not only the smash at that position but also the details of crossover, ladder, forward fast, or back fast, it will inevitably bring inconvenience to statistical work [18]. Therefore, from the perspective of representativeness and operability, the spiking mainly depends on whether the organization is a strong attack, a fast attack, or a second attack $[2,19]$. Secondly, defensively, the first is blocking. Just like serving, although there are single block, double block, and collective block, as well as various techniques and tactics, blocking is achieved more by player observation, reaction, and individual skills and tactics. Therefore, if too many blocking techniques and tactics are considered, this will inevitably bring more workload to the statistical work, but the information provided to the coaches is very limited. Therefore, the overall effect of blocking is considered. Second, the implementation of the defensive skills and tactics of the team after the block is determined by the opponent's serve, spike, block, or pass; and preventing the ball coming from the opponent mainly depends on the final defensive effect, regardless of which defensive formation or defensive tactics are used. Therefore, according to the opponent's different offensive styles, our defensive skills and tactics can be divided into receiving the serve, receiving the spiking, receiving the block and returning the ball, and receiving the pass [6].

2.2. Artificial Neural Network. As an intelligent algorithm, artificial neural network is used in a variety of fields. This research maps the collected data to $[-1,1]$ to unify each unit. The fuzzy processing is as follows [20]: 


$$
\begin{aligned}
& z_{i j}=\frac{\max \left\{S_{i j}\right\}-S_{i j}}{\max \left\{S_{i j}\right\}-\max \left\{S_{i j}\right\}}, \\
& S_{i j}=\frac{x_{i j}-\bar{x}}{S_{i}}, \\
& \bar{x}_{j}=\frac{1}{n} \sum_{i=1}^{n} x_{i j}, \\
& S_{j}^{2}=\frac{1}{n-1} \sum_{i=1}^{n}\left(x_{i j}-\bar{x}\right)^{2},
\end{aligned}
$$

where $z_{i j}$ is the measured value of the $j$-th index in the $i$-th sample and $x_{i j}$ is the measured value after normalization:

$$
[\mathrm{pn}, \text { meanp, stdp }]=\operatorname{prestd}(p) \text {. }
$$

After vectorization, the processed vector is represented by $\mathrm{pn}$, the mean value of the input vector is represented by meanp, and the deviation is represented by the vector stdp. $p$ is a set of vectors. The algorithm executed is

$$
\mathrm{pn}=\frac{(p-\text { meanp })}{\operatorname{stdp}} \text {. }
$$

\subsection{Selection of Related Functions of Matlab Neural Network}

(1) Training function

The trainlm function: its main function is to use the LM rule to train the forward network [21]. The format is

$\left[W_{1}, b_{1}, W_{2}, b_{2}, t e, t r\right]=\operatorname{trainlm}\left(W_{1}, b_{1}, W_{2}, b_{2}, f_{2}, P, T, t p\right)$.

In the above formula, $W_{1}, b_{1}, W_{2}, b_{2}$ is the initial connection weight and closed value matrix of the neural network [22, 23].

(2) Activation function

The purelin linear function format is

$$
A=\operatorname{purelin}(N) \text {. }
$$

In the above formula, $A$ is the output matrix of the network layer. The tangent sigmoid activation function tansig is a differentiable function, which is compatible with the training requirements of the BP algorithm [24]. The format is

$$
A=\operatorname{tansig}(N) \text {. }
$$

In the above formula, $N$ is the input vector. The function expression is

$$
a(n)=\tan \operatorname{sig}(n)=n .
$$

Suppose that the object of diagnosis of Chinese volleyball skills and tactics is $\mathrm{N}$ (i.e., any game in China), and the set of diagnostic criteria for $\mathrm{N}$ is

$$
I=\left\{I_{1}, I_{2}, I_{3}, \ldots, I_{n}\right\}
$$

The matter-element $R 1$ that establishes the diagnostic criteria of volleyball skills and tactics is as follows:

$$
R 1=\left[\begin{array}{ccc}
N & c_{1} & v_{1} \\
\cdots & \cdots & \cdots \\
N_{k} & c_{k} & v_{k}
\end{array}\right] .
$$

Select a match of China's A-level women's volleyball match to construct the object to be diagnosed $R 2$ :

$$
R 2=\left[\begin{array}{ccc}
N & c_{k+1} & v_{k+1} \\
\cdots & \cdots & \cdots \\
N_{n} & c_{n} & v_{n}
\end{array}\right] .
$$

The correlation function between the technical and tactical standard matter-element and the description matter-element is calculated by the following formula, that is, the relationship value between the game to be diagnosed, training, and the diagnostic criteria. Here, this value $(\lambda)$ is called proximity [25].

$$
K_{j}(k)=\left\{\begin{array}{l}
\frac{\rho\left(x, X_{o j}\right)}{\left|X_{o j}\right|}, \\
\frac{\rho\left(x, X_{o j}\right)}{\rho\left(x, X_{j}\right)-\rho\left(x, X_{o j}\right)} .
\end{array}\right.
$$

(5) Calculate the proximity of the object element to be diagnosed to the standard object element, and determine the improvement and strategy of object $\mathrm{N}$ according to the value of $a$. Among them, $\lambda \geq 0$, and the larger the value is, the more characteristic attributes of the technical and tactical index are from the standard object and the smaller the gap. If $\lambda \leq 0$, the smaller the value is, the more the technical and tactical index is away from the standard. The larger the gap, the more urgent it is to solve this problem, formulate an exact implementation plan, and make up for the mistakes in tactics and strategies. The MATLAB neural network model is shown in Figure 1.

$$
\lambda\left(I_{i}(N)\right)=\sum_{j=1}^{k_{i}} a_{i j} K_{i j}(i=1,2, \ldots, n) .
$$

\section{Volleyball Skills and Tactics Analysis Diagnostic Model Experiment}

\subsection{BP Neural Network Structure Design}

3.1.1. Determination of Input and Output Layer Parameters. This research is based on the established volleyball skill evaluation index system. The input vector is the quantitative value of the volleyball skill evaluation index, and the output 


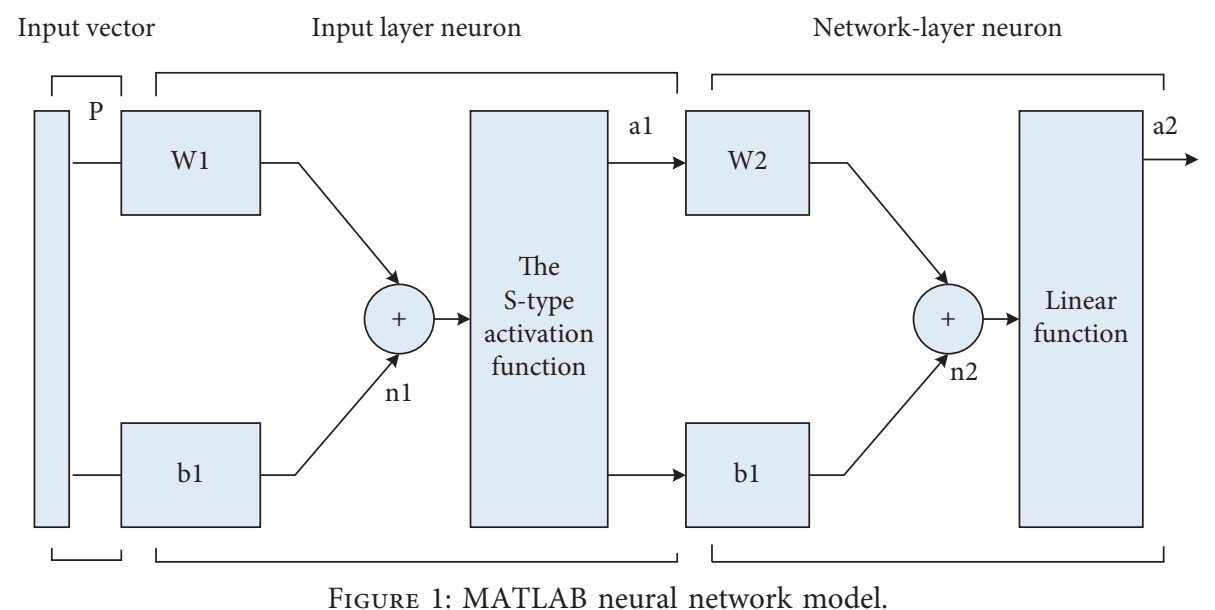

vector is the corresponding volleyball expert technical evaluation result. The numbers of input nodes of the 4 volleyball technical evaluation network models to be established are 4, 5, 4, and 4, and the number of output nodes is 1 , which is the result of the corresponding expert technical evaluation.

\subsubsection{Determination of the Number of Hidden Layer Nodes.} There is no definite and unified formula for determining the number of hidden nodes in the BP neural network, and some commonly used hidden node number estimation experiences provided by trial-and-error method can be used [26]. This study roughly estimated the number of hidden nodes by consulting the relevant literature of BP neural network application and combining the empirical formula provided by trial-and-error method. On this basis, it gradually decreases and increases. Through repeated training and comparison, it is finally determined that the number of hidden nodes that minimize the network error is 4 .

3.1.3. Setting of the Initial Weight of BP Network. The initial weights of the BP neural network for the evaluation of volleyball skills and tactics in this study are random numbers $(-0.5,0.5)$.

3.1.4. Determination of BP Network Learning Time. In the case where other conditions are unchanged, in order to obtain a good generalization ability, there is an optimal number of training times in the network. Through repeated training and comparison of input samples, it is finally determined that the maximum number of training times of BP network for volleyball technical and tactical evaluation is 32. The artificial BP neural network process is shown in Figure 2.

3.2. Steps of Applying BP Neural Network to Volleyball Technical Evaluation. The purpose of this research is to take the evaluation scores of volleyball experts on students' volleyball skills and the data of evaluation indicators of each

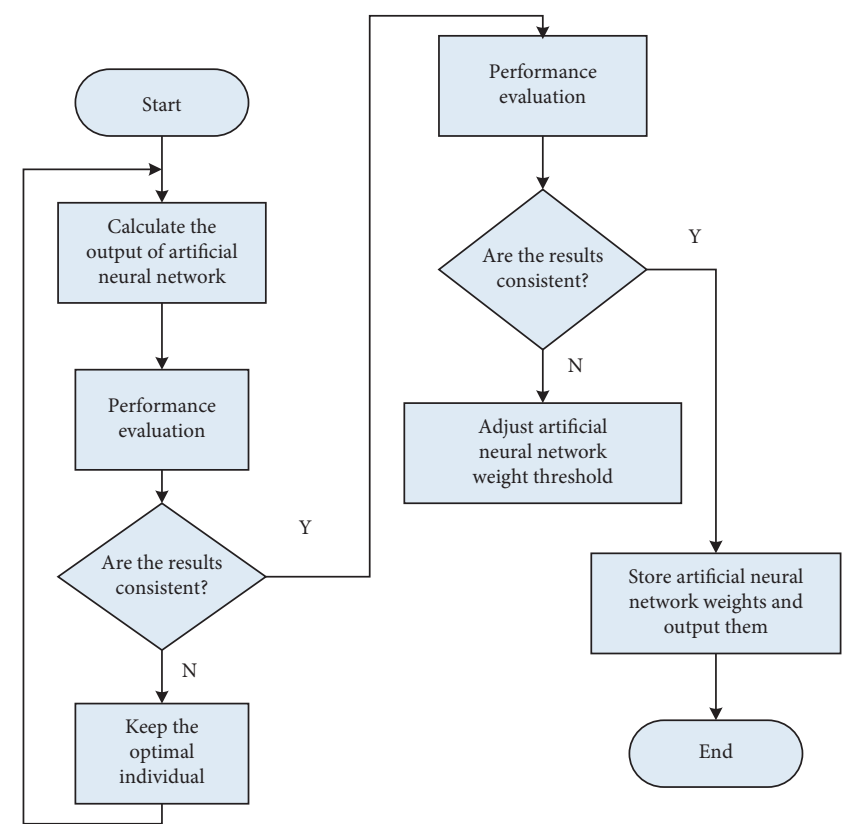

FIGURE 2: Flow of artificial BP neural network.

technical link as learning samples and analyze the relationship between the technical evaluation experience of volleyball experts and each technical link of volleyball through BP neural network and establish a basis evaluation model of volleyball skills and tactics based on BP neural network. Finally, the simulation of the technical evaluation thinking of volleyball experts is realized, so that young teachers can successfully predict the technical evaluation results of experts through the model.

According to research needs, four BP neural network models for volleyball serve, pad, pass, and buckle will be established. The first layer is the input layer, and the input variables are the evaluation indicators of the volleyball technique; the middle is the hidden layer, which adjusts the weights of neurons to make the network approach the result of the volleyball expert's evaluation of the technique; the last layer is the output layer, outputting the actual evaluation results of volleyball experts on the technology. Specific steps are as follows: 
(1) Sample selection and pretreatment: According to the research objectives, the expert technical evaluation results of 20 volleyball students are selected as training samples; the expert technical evaluation results of the remaining 10 volleyball general students are used as test samples.

(2) Selection of relevant indicators: In a volleyball game, computer analysis mainly includes two methods: technical analysis and tactical analysis. Technical analysis mainly starts from the game video and processes the video. The single sword situation can be analyzed separately, fast or full, for the coach to analyze the basic situation of the athletes in order to formulate the corresponding training plan. The tactical analysis is mainly to analyze a large amount of data content, including the scores of various technical and tactical items, the loss of points, and the actual utilization. By combining with the game video, a comprehensive analysis of the players is carried out. Take the volleyball game as an example. These technical and tactical items mainly include offense, defense, counterattack, and preparation actions. This is also the main indicator selected. Corresponding to the score loss of each part of the body, three-dimensional all-round statistics can be obtained.

(3) Design of input and output parameters: The national volleyball team participates in a large number of games every year, and there are a large number of game videos and a big amount of game data. Use this software's video editing function to edit the video and generate a large number of video clips and game statistics. Through the video retrieval option, select the desired clip. The input quantity must be selected from variables that are not highly correlated and have a great influence on the output quantity. The output quantity represents the function to be realized by the system. Normally, it is necessary to perform certain processing on the input variables, such as signal processing, before it can be used as the input of the neural network. By observing the training situation of the volleyball team players, 12 technical and tactical indicators were extracted: a1, offensive utilization rate; a2, offensive scoring rate; $\mathrm{a}$, offensive scoring rate; b1, defensive utilization rate; $b 2$, defensive scoring rate; b3, defensive scoring rate; $\mathrm{cl}$, counterattack utilization rate; c2, counterattack scoring rate; c3, counterattack loss rate; $\mathrm{d} 1$, preparatory action usage rate; $\mathrm{d} 2$, preparatory action scoring rate; and $\mathrm{d} 3$, preparatory action scoring rate. Selecting these 12 technical and tactical indicators as input nodes, the system wants to predict the victory of the game, so the output layer node is set as the probability of winning the game. There is 1 output layer. Use the powerful self-learning function of neural network. Establish a prediction model for athletes' competition wins.
(4) The design of training sample set: Pay attention to the balance of the samples, and cross-input different types of samples. Generally, the collected samples are divided into two parts: one for training the network and the other for verification. Look at the error of the training sample to test the generalization ability of the network. This research uses the competition data of the world's outstanding volleyball team players collected by the "National Volleyball Team Information Platform" as a training sample. Due to the obvious differences in the size and unit of the original test data, in order to reduce or even eliminate the effect of small indicators of the model, before the training of the neural network, it is necessary to unify the metric standards of each indicator of the training sample.

3.3. BP Network Evaluation Model Design. This research uses the relevant functions in the MATLAB neural network toolbox to realize the construction, training, and testing of the neural network model for the evaluation of volleyball technology. The volleyball technology evaluation network model in this study is a three-layer BP neural network with a hidden layer. The input vector $P$ is a 4-dimensional and 5dimensional vector, and the output vector $t$ is a one-dimensional vector. Use the random initialization function initf to initialize the weight and closed value matrix of the network input layer to the hidden layer and the hidden layer to the output layer, and set the expected error index err_goal, the maximum number of training steps max_epoch, and the network learning rate $1 \mathrm{r}$. The postprocessing function postreg is used to perform linear regression analysis on the output and prediction results to test the generalization ability and accuracy of the BP network.

(1) Create and initialize the BP neural network model.

(2) Read and process the training data and prediction data of the home team, and then normalize the processed data. Only then can the data be used as the input of the BP neural network.

(3) Read from the database and set the learning rate and maximum learning times.

(4) Read and set the error accuracy from the database and use the predicted data of the home team to make predictions to get the home team's victory index.

In the training process of the volleyball technique and tactics evaluation neural network, the sample data of the volleyball technique evaluation index is repeatedly simulated and learned, and finally the network parameters with the smallest error and the highest accuracy are saved as the network model for subsequent verification and evaluation. The weight and closed value initialization matrix are saved as the file in it BP:mat, and the final weight and closed value matrix obtained by network training are saved as the file net BP:mat. These documents will provide necessary parameter support for the subsequent instance verification of the volleyball technical and tactical evaluation model. 


\section{Volleyball Skills and Diagnosis Model}

4.1. BP Network Test Verification and Result Analysis. When analyzing the effects of volleyball techniques and tactics, it is necessary not only to conduct a horizontal analysis for yourself but also to compare and analyze the average technical and tactical use of other teams in a longitudinal direction, that is, to compare the use of a certain technique and tactics of the team with the use of other teams. The effect is compared, so that we can more clearly and directly see the level of the team's technology in each team, what the advantages are, and what the shortcomings are. Therefore, when analyzing and evaluating a certain technical and tactical level or multiple technical and tactical levels of volleyball, it is necessary to comprehensively consider the level of the evaluated object or various physical qualities to establish a volleyball team equivalent to the technical level. Reference value rating model for coaches is used to refer to before, during, and after the game. BP network serve evaluation results are shown in Table 1. BP network serve evaluation and analysis results are shown in Figure 3. It can be seen that the average difference between the evaluation scores output by the network and the scores of the actual experts is less than $1 \%$, reaching a very high accuracy, indicating that the volleyball skill evaluation model based on the BP neural network is technically feasible, and the results are relatively reliable.

The evaluation results of BP network pad ball are shown in Table 2. The BP network pad ball evaluation and analysis results are shown in Figure 4. It can be seen from Table 2 that the number of fields participating in the sample back-substitution test and the percentages of the same-inverse two factors (i.e., success rate and failure rate) are basically normal distribution at each level, which is basically consistent with the theoretical division results. It is shown that the evaluation standards formulated in this section for the comprehensive evaluation of offensive and defensive techniques and tactics have certain validity. Therefore, it can be considered that the same-contrary evaluation model developed in this study for evaluating the effects of the comprehensive application of offensive and defensive techniques and tactics has certain validity and reference. The coaches serve as a reference to a certain extent when making a comprehensive evaluation of the team's offensive and defensive skills and tactics in training or competition. The successful (same) effect of Chinese volleyball players in the comprehensive use of offensive and defensive skills and tactics has almost reached the world's excellent level, and the effect of turnover (reverse) is at the world average level. It is shown that Chinese volleyball players still have room for improvement in the use of offensive and defensive skills and tactics in the game. Coaches are requested to pay attention to them in usual training and competitions, especially to minimize the mistakes in offensive and defensive skills and tactics and to improve offensive and defensive skills. At the same time, we observed from Table 2 that the level of successful skills and tactics of the top 8 world volleyball players is mostly concentrated in the good, medium, and poor levels, and the middle, poor, and poor levels account for about $70 \%$. This shows that the top 8 volleyball players in the world have instability in the use of offensive and defensive skills and tactics, and there is still room for improvement. Then we observe the failure rate. From Table 2, we can see that the top 8 players in the world in the volleyball game have only about $37 \%$ of the turnovers. This shows that each team has the use of offensive and defensive techniques and tactics. There are too many mistakes. This point must arouse the attention of coaches from various countries and strengthen the training of offensive and defensive skills and tactics in order to control the mistakes to a minimum in the game.

In order to further verify the effectiveness of the neural network evaluation model of volleyball technology, we asked experts from the volleyball teaching and research section to evaluate the skills of serving, padding, passing, and spiking for 30 students in the volleyball group. At the same time, this study also used the neural network evaluation of volleyball skills. The model system evaluated and scored these 30 volleyball students for the skills of serving, padding, passing, and spiking. Then, the volleyball expert evaluation system and the computer neural network technology evaluation system are quantitatively compared, the difference between the two is analyzed using the analytic hierarchy process, and the training function of the neural network is trained. Set the number of training samples to 1000 and the number of training times to 2000; the minimum error is controlled at 0.001 and finally 7 neural networks, namely, N0-N6, are obtained. Among them, N0 is the neural network trained by the total data set, and N1-N6 is a neural network trained on data from cluster 1 to cluster 6 . When the training is completed, the player PG will be handed over to N0-N6 in order to control the real-time game and record the game data. Each neural network runs 20 games. The results of the mathematical statistics test are shown in Table 3. BP network output and actual regression analysis results are shown in Figure 5. From the above table, we can see that the difference in the evaluation scores of the four major volleyball techniques between volleyball experts and neural network technology evaluation systems has not reached statistical significance $(P>0.05)$, and the correlation coefficient $R$ is close to 1 , which shows that the volleyball technology neural network evaluation model has strong reliability and effectiveness.

4.2. Volleyball Skills and Tactics. The factors that affect the game can be roughly divided into several categories: psychological conditions, physiological conditions, technical influences, tactical influences, and so forth. They influence each other and work together on the result, and the techniques and tactics are the main influencing factors, so statistical analysis of the techniques and tactics is very useful and necessary. The weight optimization of the BP network is an unconstrained optimization problem, which establishes a connection with the VC in the background by calling the Matlab engine. Through the neural network training, it can reflect the functional relationship between the technical and tactical indicators and the game winning rate and basically establish a volleyball technical and tactical analysis and 
TABLE 1: Evaluation results of BP network service.

\begin{tabular}{lccccc}
\hline Test sample & Practical expert rating & Network output & Absolute error & Relative error (\%) & Average relative error (\%) \\
\hline 1 & 82 & 83.28 & 1.28 & 1.56 & 0.92 \\
2 & 87 & 85.36 & 1.64 & 1.88 \\
3 & 88 & 87.58 & 0.42 & 0.48 \\
4 & 70 & 69.90 & 0.10 & 0.14 \\
5 & 79 & 79.89 & 0.89 & 1.13 \\
6 & 75 & 75.58 & 0.58 & 0.77 \\
7 & 78 & 77.83 & -0.17 & 0.22 \\
8 & 82 & 83.14 & 1.14 & 1.39 \\
9 & 70.57 & 0.43 & 0.61 \\
10 & 71 & 60.63 & 0.63 & 1.05 \\
\hline
\end{tabular}

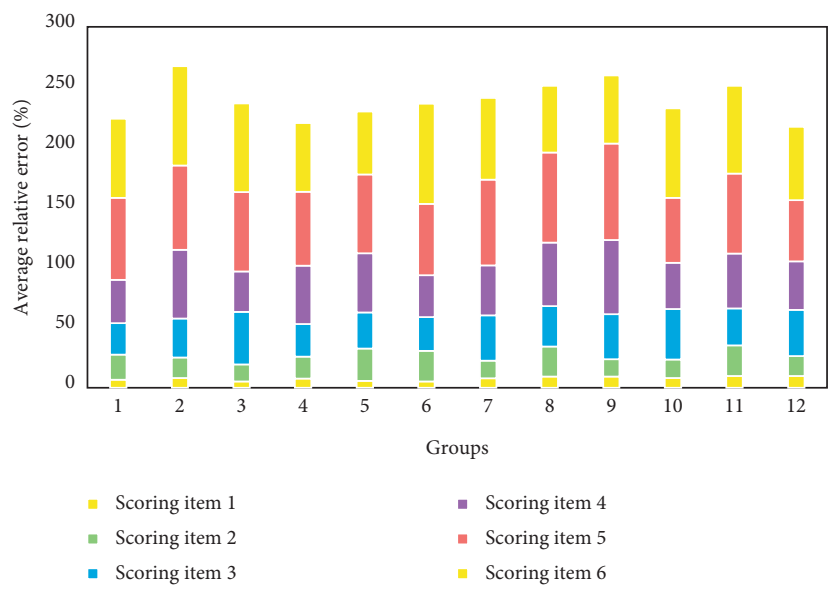

FIgURE 3: Analysis results of BP network service evaluation.

TABLE 2: Evaluation results of BP network padding.

\begin{tabular}{lccccc}
\hline Test sample & Practical expert rating & Network output & Absolute error & Relative error (\%) & Average relative error (\%) \\
\hline 1 & 81 & 80.57 & -0.43 & 0.53 & 0.12 \\
2 & 78 & 78.09 & 0.09 & 0.38 \\
3 & 85 & 85.32 & 0.32 & 0.30 & 0.59 \\
4 \\
5 & 76 & 75.77 & -0.23 & 0.32 & 0.48 \\
6 & 82 & 82.48 & 0.48 & 0.82 & 0.49 \\
7 & 78 & 78.25 & 0.25 & 0.26 \\
8 & 77 & 77.63 & 0.63 & 0.97 \\
9 & 80 & 79.61 & -0.39 & -0.19 & \\
10 & 73 & 72.81 & 0.58 & & \\
\hline
\end{tabular}

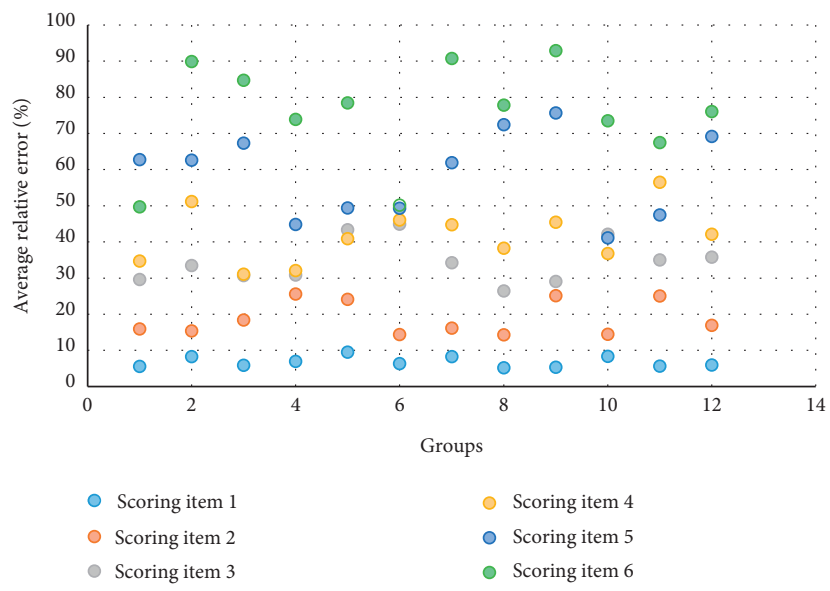

FIGURE 4: Analysis results of BP network ball cushion evaluation. 
TABLE 3: Test results of mathematical statistics.

\begin{tabular}{lcccc}
\hline $\begin{array}{c}\text { Average relative error of } \\
\text { volleyball expert and } \\
\text { network evaluation } \\
\text { model score (\%) }\end{array}$ & $T$ & $R$ & $P$ \\
\hline Serve & 0.62 & 1.156 & 0.9221 & 0.089 \\
Pad the ball & 0.47 & 0.793 & 0.9854 & 0.103 \\
Pass & 0.49 & 0.815 & 0.9718 & 0.0925 \\
Spike & 0.56 & 1.027 & 0.9433 & 0.081 \\
\hline
\end{tabular}

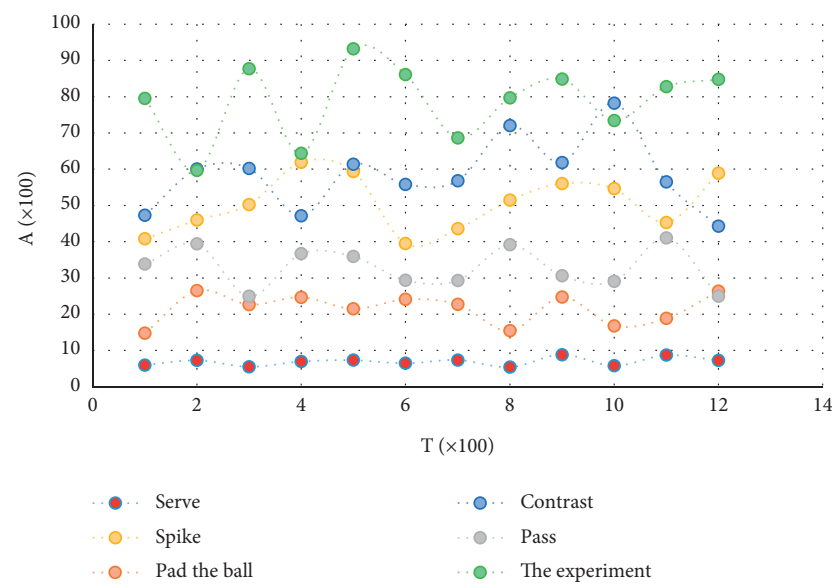

FIGURE 5: BP network output and actual regression analysis results.

prediction model. Click "Predicted Win Rate" to analyze the probability of winning against your opponent. Technical and tactical indicators are shown in Table 4. The technical and tactical index analysis results are shown in Figure 6. It can be seen from Table 4 that the overall prediction rate is $60.9 \%$.

Generally speaking, every sports team wants to give full play to the team's skills in every game to get a better evaluation. In this regard, we can use SPA to evaluate multiple or single technical indicators. For example, a volleyball team spikes $100(\mathrm{~N})$ times in a game, succeeds (4 points) 45 times (a), makes mistakes (out of bounds, stops 1 point) 12 times (c), and the remaining 43 times (b). Success and failure cannot be determined. BP network pass evaluation results are shown in Table 5. BP network pass evaluation and analysis results are shown in Figure 7. It can be seen from Table 5 that when $a>b>c$, the application effect of a certain technology is excellent. Comparing the expressions of the Chinese team's eight technical and tactical similarities, differences, and counters, it can be seen that, in terms of offensive technology and tactics, the technical effect of the serve is in the counter-trend zone, counter-trend level 3 , and global level 12 in the similarity, difference, and counter-posture ranking table of the set pair analysis connection degree. The strong attack effect is in the same potential area, the same potential level 1 , and the global level 1. Judging from the above analysis of offensive techniques and tactics, the offensive tactical indicators reflect the better use of techniques and tactics, while the effects of serving techniques are slightly insufficient. Looking at the defensive technique again, the effect of the blocking technique belongs to the counter-trend zone, counter-trend level 3, and the overall 12 rounds; the technical effects of receiving and serving belong to the same level, the world belongs to level 1 , both receiving and serving belong to level 5 , and the overall level is 14; the technical effect of catching and returning the ball belongs to the counter-trend zone, counter-trend level 5 , and overall level 14. From the defensive analysis, the technical effects of the other three indicators are not ideal except for the ideal receiving and serving effect. Especially in the application of the technology of catching and returning the ball, the degrees of similarity, difference, and contradiction are $a<c, a<b$, and $b<c$; that is, the success rate is less than the error rate, and the error rate is too large, indicating that the application of these two technologies needs to be greatly improved, which deserves more attention from our coaches and athletes in the future. Strengthen the targeted training of catching and returning the ball to keep the turnover rate to a minimum.

Figure 8 shows the results of the technical and tactical analysis of the 10 games. The BP network spiking evaluation results are shown in Table 6 . In contrast, from the test results of the same-inverse data on the technical and tactical indicators of the selected 10 games, the test results of the sameinverse data of the independent use of the technical and tactical indicators in the 16 games that participated in the back-testing are compared with the theoretically divided number of fields and percentages. The results are basically the same. It is shown that the evaluation standard of this evaluation model has certain validity. Therefore, it can be considered that the same-counter-level evaluation model of the independent use of techniques and tactics developed in this research has certain practicality and reference value. Below we use the level evaluation model of the independent application effect of offensive and defensive technical and tactical indicators developed in this section as a reference basis and compare and analyze the specific application of offensive and defensive techniques and tactics in the Chinese women's volleyball finals. By referring to the model, it can be seen that the Chinese team's serve is of the same technique; that is, the scoring rate is an excellent level, and the opposite technique, that is, the turnover rate, is also an excellent level; the strong attack and the opposite value are both good; the fast attack and the same technique and tactics are general, and the opposite skills and tactics are excellent. From the reflection of several offensive technical and tactical indicators, the overall offensive situation of the Chinese women's volleyball team in this game is relatively ideal. The application effect of serving technology has reached the highest level in the world, and the strong attack is of a good level. This shows that the application effect of the Chinese women's volleyball team has reached a higher level in the world in this game. Although the offensive effect, that is, the success rate, is average in the fast break, there are fewer mistakes. There are some unsatisfactory uses of fast break, which are closely related to the requirements of the first pass, second pass, and the degree of tacit cooperation between the players. This provides us with some information, telling us that we should strengthen fast break training in future training. Not only the first pass but also the second pass can 
TABLE 4: Technical and tactical indicators.

\begin{tabular}{lccccc}
\hline Technology & Have to & Loss & Success rate & Technology ratio & Winning percentage is predicted \\
\hline \multirow{2}{*}{ Attacking } & 35 & 13 & 80.2 & 79.8 & \\
Defense & 4 & 1 & 83.3 & 45 & 60.9 \\
Prepare for action attack & 2 & 5 & 50 & 48 & \\
\hline
\end{tabular}

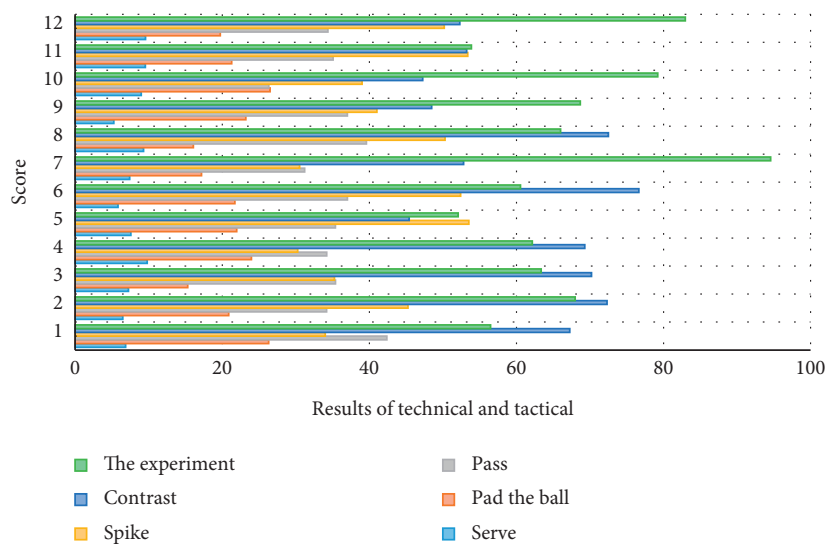

Figure 6: Analysis results of technical and tactical indicators.

TABLE 5: Evaluation results of BP network pass.

\begin{tabular}{|c|c|c|c|c|c|}
\hline Test sample & Practical expert rating & Network output & Absolute error & Relative error (\%) & Average relative error $(\%)$ \\
\hline 1 & 82 & 81.63 & -0.37 & 0.45 & \multirow{10}{*}{0.57} \\
\hline 2 & 78 & 79.02 & 1.02 & 1.31 & \\
\hline 3 & 78 & 78.39 & 0.39 & 0.50 & \\
\hline 4 & 68 & 67.55 & -0.45 & 0.66 & \\
\hline 5 & 80 & 79.86 & -0.14 & 0.18 & \\
\hline 6 & 80 & 80.25 & 0.25 & 0.31 & \\
\hline 7 & 75 & 74.61 & -0.39 & 0.52 & \\
\hline 8 & 81 & 81.35 & 0.35 & 0.43 & \\
\hline 9 & 72 & 71.44 & -0.56 & 0.78 & \\
\hline 10 & 70 & 70.39 & 0.39 & 0.56 & \\
\hline
\end{tabular}

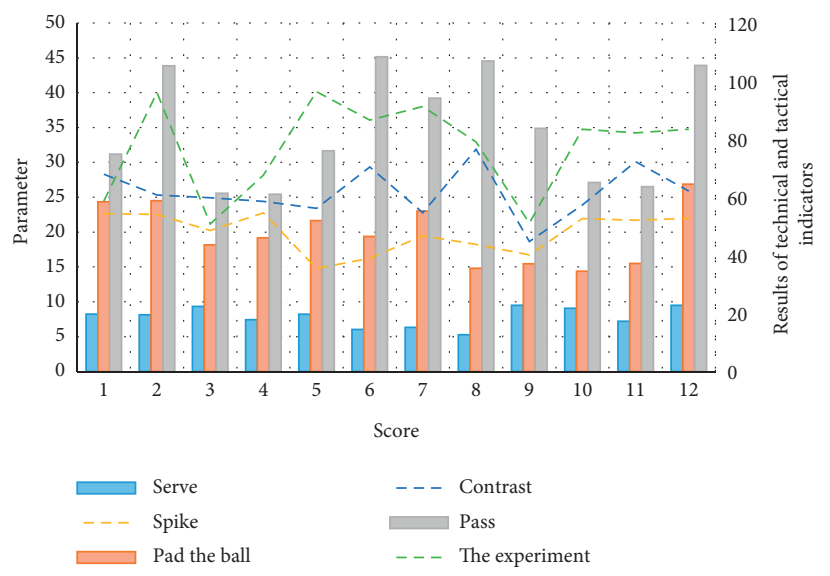

FIGURE 7: Analysis results of BP network pass evaluation. 


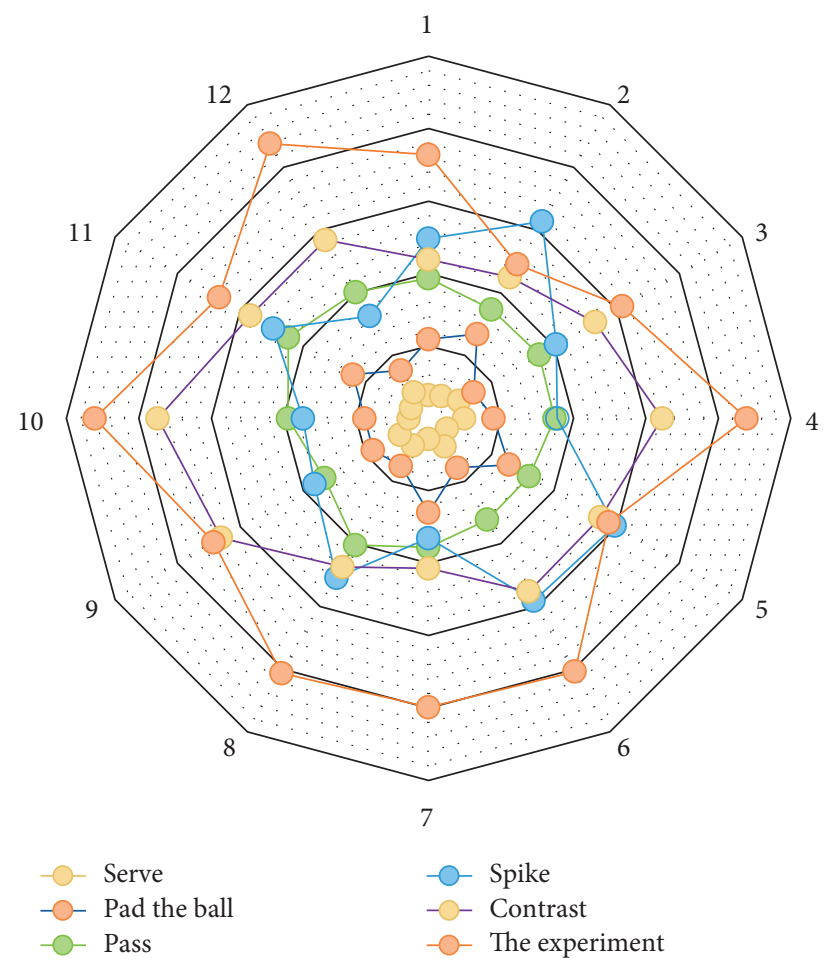

FIGURE 8: 10 technical and tactical analysis results of the competition.

TABLE 6: BP network spike evaluation results.

\begin{tabular}{lccccc}
\hline Test sample & Practical expert rating & Network output & Absolute error & Relative error (\%) & Average relative error (\%) \\
\hline 1 & 82 & 82.66 & 0.66 & 0.80 & 0.53 \\
2 & 79 & 79.42 & 0.42 & 0.38 \\
3 & 85 & 85.32 & 0.32 & 1.27 & 0.90 \\
4 \\
5 & 70 & 69.11 & -0.89 & 0.56 & 0.69 \\
6 & 83 & 83.75 & 0.75 & 1.03 \\
7 & 80 & 79.65 & -0.45 & 0.42 & \\
8 & 76 & 75.22 & -0.78 & 0.61 \\
9 & 79 & 78.67 & -0.33 & 0.37 \\
10 & 77 & 76.53 & -0.47 & 0.25 & \\
\hline
\end{tabular}

organize the fast break, and the fast break can also be used when the first pass and the second pass are slightly off. At the same time, the targeted training of the setter and the cooperation training between the players should be strengthened. While ensuring the fast break, it also emphasizes the quality of the fast break and the effect achieved, so that the Chinese women's volleyball team can be used in various offensive techniques and tactics. The application has reached the highest level in the world.

\section{Conclusion}

In a volleyball game, computer analysis mainly includes two methods: technical analysis and tactical analysis. Technical analysis mainly starts from the game video and processes the video. The single sword situation can be analyzed separately, fast or full, for the coach to analyze the basic situation of the athletes in order to formulate the corresponding training plan. By combining with the game video, a comprehensive analysis of the players is carried out. Take the volleyball game as an example. These technical and tactical items mainly include offense, defense, counterattack, and preparation actions. This is also the main indicator selected. Corresponding to the score loss of each part of the body, threedimensional all-round statistics can be obtained.

The tactical analysis is mainly to analyze a large amount of data content, including the scores of various technical and tactical items, the loss of points, and the actual utilization. The national volleyball team participates in a large number of games every year, and there are a large number of game videos and a big amount of game data. Use this software's video editing function to edit the video and generate a large number of video clips and game statistics. Through the video retrieval option, select the desired clip. The input quantity must be selected from variables that are not highly correlated and have a great influence on the output quantity. The 
output quantity represents the function to be realized by the system.

Pay attention to the balance of the samples, and crossinput different types of samples. Generally, the collected samples are divided into two parts: one for training the network and the other for verification. Look at the error of the training sample to test the generalization ability of the network. This research uses the competition data of the world's outstanding volleyball team players collected by the "National Volleyball Team Information Platform" as a training sample. Due to the obvious differences in the size and unit of the original test data, in order to reduce or even eliminate the effect of small indicators of the model, before the training of the neural network, it is necessary to unify the metric standards of each indicator of the training sample.

\section{Data Availability}

No data were used to support this study.

\section{Conflicts of Interest}

The authors declare that there are no conflicts of interest with any financial organizations regarding the material reported in this manuscript.

\section{References}

[1] Y. Zhao, H. Li, S. Wan et al., "Knowledge-aided convolutional neural network for small organ segmentation," IEEE journal of biomedical and health informatics, vol. 23, no. 4, pp. 1363-1373, 2019.

[2] A. Y. Alanis and Y. Alma, "Electricity prices forecasting using artificial neural networks," IEEE Latin America Transactions, vol. 16, no. 1, pp. 105-111, 2018.

[3] J. Daniel, A. Abudhahir, and J. J. Paulin, "Magnetic flux leakage (MFL) based defect characterization of steam generator tubes using artificial neural networks," Journal of Magnetics, vol. 22, no. 1, pp. 34-42, 2017.

[4] D. Carvalho, N. A. Garcia-Martinez, J. L. Lado et al., "Realspace mapping of topological invariants using artificial neural networks," Physical Review B, vol. 97, no. 11, pp. 115453.1-115453.8, 2018.

[5] A. Pandey and A. Mishra, "Application of artificial neural networks in yield prediction of potato crop," Russian Agricultural Sciences, vol. 43, no. 3, pp. 266-272, 2017.

[6] G. Carleo and M. Troyer, "Solving the quantum many-body problem with artificial neural networks," Ence, vol. 355, no. 6325, pp. 602-606, 2016.

[7] S. Tiryaki, z. ahinükrü, and A. Aydn, "Employing artificial neural networks for minimizing surface roughness and power consumption in abrasive machining of wood," European Journal of Wood \& Wood Products, vol. 75, no. 3, pp. 1-12, 2017.

[8] M. Adavi, M. Salehi, and M. Roudbari, "Artificial neural networks versus bivariate logistic regression in prediction diagnosis of patients with hypertension and diabetes," Medical Journal of the Islamic Republic of Iran, vol. 30, no. 1, 312 pages, 2016.

[9] A. Sedaghat, H. Bayat, and A. A. Safari Sinegani, "Estimation of soil saturated hydraulic conductivity by artificial neural networks ensemble in smectitic soils," Eurasian Soil Science, vol. 49, no. 3, pp. 347-357, 2016.

[10] X. Yu and X. Guo, "Hourly photosynthetically active radiation estimation in Midwestern United States from artificial neural networks and conventional regressions models," International Journal of Biometeorology, vol. 60, no. 8, pp. 1247-1259, 2016.

[11] F. Leiva-Villacorta, A. Vargas-Nordcbeck, and D. H. Timm, "Non-destructive evaluation of sustainable pavement technologies using artificial neural networks," International Journal of Pavement Research and Technology, vol. 10, no. 2, pp. 139-147, 2016.

[12] H. Mahmoud, "Using artificial neural networks to forecast economic impact of multi-hazard hurricane-based events," Sustainable \& Resilient Infrastructure, vol. 1, no. 1-2, pp. 63-83, 2016.

[13] G. Vicario, G. Craparotta, and G. Pistone, "Meta-models in computer experiments: kriging versus artificial neural networks," Quality and Reliability Engineering International, vol. 32, no. 6, pp. 2055-2065, 2016.

[14] J. M. Lopez-Guede, J. A. Ramos-Hernanz, E. Zulueta, U. Fernadez-Gamiz, and F. Oterino, "Systematic modeling of photovoltaic modules based on artificial neural networks," International Journal of Hydrogen Energy, vol. 41, no. 29, pp. 12672-12687, 2016.

[15] A. Lino, Á. Rocha, and A. Sizo, "Virtual teaching and learning environments: automatic evaluation with artificial neural networks," Cluster Computing, vol. 22, no. 4, pp. 1-11, 2017.

[16] L. G. Esteban, P. De Palacios, M. Conde, F. G. Fernández, A. García-Iruela, and M. González-Alonso, "Application of artificial neural networks as a predictive method to differentiate the wood of Pinus sylvestris L. and Pinus nigra Arn subsp. salzmannii (Dunal) Franco," Wood Science and Technology, vol. 51, no. 5, pp. 1249-1258, 2017.

[17] N. R. Secco and B. S. d. Mattos, "Artificial neural networks to predict aerodynamic coefficients of transport airplanes," Aircraft Engineering \& Aerospace Technology, vol. 89, no. 2, pp. 211-230, 2017.

[18] E. Yllmaz and A. Ersen, "Fetal state assessment from cardiotocogram data using artificial neural networks," Journal of Medical and Biological Engineering, vol. 36, no. 6, pp. 820832, 2016.

[19] W.-E. Hsu, Y.-H. Chang, Y.-J. Huang, J.-C. Huang, and C.-T. Lin, "A pH/light dual-modal sensing ISFET assisted by artificial neural networks,” ECS Transactions, vol. 89, no. 6, pp. 31-37, 2019.

[20] P. E. Rauber, S. G. Fadel, A. X. Falcão et al., "Visualizing the hidden activity of artificial neural networks," IEEE Transactions on Visualization and Computer Graphics, vol. 23, no. 1, pp. 101-110, 2016.

[21] Y. Gong, Y. Zhang, S. Lan, and H. Wang, "A comparative study of artificial neural networks, support vector machines and adaptive neuro fuzzy inference system for forecasting groundwater levels near lake okeechobee, Florida," Water Resources Management, vol. 30, no. 1, pp. 375-391, 2016.

[22] S. Li, M. Fairbank, C. Johnson et al., "Artificial neural networks for control of a grid-connected rectifier/inverter under disturbance, dynamic and power converter switching conditions," IEEE Transactions on Neural Networks and Learning Systems, vol. 25, no. 4, pp. 738-750, 2017.

[23] M. Safa, S. Samarasinghe, and M. Nejat, "Prediction of wheat production using artificial neural networks and investigating indirect factors affecting it: case study in canterbury province, 
New Zealand," Journal of Agricultural Science and Technology A, vol. 17, no. 4, pp. 791-803, 2018.

[24] T.-H. Sun, F.-C. Tien, F.-C. Tien, and R.-J. Kuo, “Automated thermal fuse inspection using machine vision and artificial neural networks," Journal of Intelligent Manufacturing, vol. 27, no. 3, pp. 639-651, 2016.

[25] A. Arnaiz-González, F. Valdivielso, A. Bustillo et al., "Using artificial neural networks for the prediction of dimensional error on inclined surfaces manufactured by ball-end milling," International Journal of Advanced Manufacturing Technology, vol. 83, no. 5-8, pp. 847-859, 2016.

[26] L. Wu, Q. Zhang, C.-H. Chen, K. Guo, and D. Wang, "Deep learning techniques for community detection in social networks," IEEE Access, vol. 8, no. 8, pp. 96016-96026, 2020. 